\title{
EN TORNO DE LA TEORIA DE ALF ROSS
}

\author{
EDUARDO GARCÍA MÁYNEZ \\ Universidad Nacional \\ Autónoma de México
}

En el presente artículo me propongo discutir la concepción rossiana del derecho. Siguiendo el orden de la obra On Law and Justice (Stevens \& Sons, Ltd. London, 1958), haré primeramente referencia a la teoría de Ross sobre los enunciados lingüísticos. El jurista danés distingue: a) aserciones, o enunciados de significación representativa; b) exclamaciones, que no tienen significado representativo ni pretenden influir sobre la conducta ajena; c) directivas, o enunciados no representativos que responden al propósito de ejercer influjo sobre el comportamiento de otros sujetos.

De los tres miembros de esta clasificación nos interesa, por ahora, detenernos en el último. Ross engloba bajo el término común directivas un conjunto sumamente heterogéneo de enunciados no representativos: mandatos, súplicas, peticiones, sugerencias, normas y deseos. Este punto es el primero que juzgamos criticable. Por un lado, parece poco feliz referir la expresión genérica enunciados a normas de conducta. Ello, en efecto, tiende a insinuar, desde un principio, que los juicios normativos, lejos de imponer deberes $\mathrm{u}$ otorgar derechos, expresan simplemente el deseo de mover a otro a que se conduzca de tal o cual manera, no porque debe conducirse así, o tenga el derecho de hacerlo, sino porque quien emite el juicio quiere orientar en tal o cual sentido el comportamiento ajeno. De esta suerte se empieza por negar, sin razón suficiente, la existencia de lo normativo - conservando sólo la palabra - y se coloca a las normas en el mismo plano y al mismo nivel, no solamente de los mandatos - cosa que muchos han hecho- sino de las sú- 
plicas, las peticiones, las sugerencias y los deseos. Tal asimilación es censurable, pues no existen fundamentos para sostener que cuando el legislador dicta una ley o un tribunal emite una sentencia, hacen algo que pueda equipararse a la formulación de un deseo o a la expresión de un ruego. Lo que, en cierto modo al menos, disfraza el error de Ross, es el uso del término común directivas, pues no hay duda de que tanto un precepto legal como una sentencia encierran la pretensión de dirigir o regular la conducta humana. Pero las llamadas directivas difieren de manera radical cuando tenemos que vérnoslas con normas y cuando únicamente se trata de sugerencias o invitaciones. Por lo demás, la tesis tampoco debe causar extrañeza, pues una de las convicciones más arraigadas de nuestro autor es que la idea de norma es de índole metafísica $y$, por ende, totalmente inaceptable dentro de un discurso "científico".

Las dificultades empiezan cuando aquél se pregunta si las frases que aparecen en obras jurídicas -un tratado de derecho civil o derecho administrativo, por ejemplo - tienen también el carácter de directivas. La cuestión le parece dudosa, "pues entre el lenguaje usado por John Hannold en su libro Cases and Material of the Law of Sales and Sales Financing y el que se emplea en la Uniform Sales Act o en otros cuerpos de leyes, no encontramos ninguna diferencia". La diferencia - prosigue Ross - existe sin embargo, pues no hay duda de que las proposiciones que aparecen en un manual de derecho "pretenden, en cierto sentido al menos, describir, no prescribir" (pág. 11 de la traducción italiana de Gavazzi). Consideremos la cuestión un poco más despacio. Súpongase que abro un manual de derecho civil mexicano y leo las siguientes palabras: "Quien descubra un tesoro en terreno ajeno tiene derecho a conservar para sí la mitad del tesoro y está obligado a entregar la otra mitad al dueño del predio". ¿Qué carácter debemos atribuir al juicio expresado por esta oración gramatical?... ¿Trátase de un simple enunciado? ... ¿De la descripción de algo? ... ¿De una regla 
de comportamiento?... Parece clarísimo que no estamos ante un juicio descriptivo, pues las palabras "quien descubra un tesoro en terreno ajeno tiene derecho a conservar para sí la mitad del tesoro y está obligado a entregar la otra mitad al dueño del predio" no describen la conducta del sujeto que ha descubierto un tesoro ni, hipotéticamente, la de todos los que tengan esta suerte. Lo que la oración expresa es otra cosa, a saber, que si alguien encuentra un tesoro en terreno que no es suyo, el descubrimiento le dará el derecho de quedarse con la mitad del hallazgo, y le impondrá la obligación, correlativa de una facultad del dueño del predio, de entregar a éste la otra parte. El juicio expresado por la oración que estamos examinando es, pues, por un lado, atributivo de un derecho y, por otro, impositivo de un deber. No se trata, en consecuencia, de un enunciado, pues las palabras integrantes de la oración gramatical no expresan, como diría Husserl, una matter of fact, ni describen el comportamiento efectivo de los descubridores de tesoros. Ross argumenta, sin embargo, que las proposiciones que pueden leerse en los manuales o tratados jurídicos pretenden, "en cierto sentido al menos, describir, no prescribir". Lo que en realidad ocurre, en nuestra opinión, es que el jurista escandinavo confunde dos cosas que es necesario mantener separadas. Por una parte, la expresión verbal de un juicio claramente normativo $y$, por otra, la enunciación de que "de acuerdo con tal o cual precepto -en el caso, el artículo 877 del Código Civil del Distrito y Territorios Federales- 'quien descubra un tesoro en terreno ajeno tiene derecho a conservar para sí la mitad del tesoro y está obligado a entregar la otra mitad al dueño del predio'". Esta proposición sí es claramente enunciativa, o, si se quiere, descriptiva, pues lo que enuncia es que el mencionado artículo tiene tal o cual contenido. Pero una cosa es declarar que un artículo de un Código dice ésto o aquéllo, y otra, muy distinta, dar expresión a lo preceptuado. Hay un pasaje en que Ross parece darse cuenta de su error, aquél en 
que declara: "La proposición de un manual jurídico que, prima facie, tenga el carácter de una directiva $D$, debe, pues, ser entendida como proposición que no es de derecho, sino sobre el derecho, y habrá que presentarla así:

$D$ es derecho vigente (en Illinois, California, etc.)".

Si consideramos la anterior como aserción sobre el derecho y, siguiendo a Ross, le damos el nombre de $A$, al punto caeremos en la cuenta de que $A$, o sea el juicio enunciativo que afirma que " $D$ es derecho vigente (en Illinois, California, etc.) y la proposición normativa designada por $D$ son cosas completamente distintas.

Veamos ahora qué debe decirse de la explicación que hace Ross del término 'derecho vigente', y de la que cabría llamar "concepción lúdica" del derecho.

¿Puede realmente considerarse el juego de ajedrez, o cualquiera otro, como 'modelo' o 'pauta explicativa' del orden jurídico?

El primer reparo que ocurre formular es que las reglas de los juegos no pueden servir como punto de partida de una doctrina sobre lo que el derecho es, por la sencilla razón de que no pertenecen, al menos normalmente, al ámbito de lo jurídico, sino al de los convencionalismos sociales. La circunstancia de que algunas veces el legislador las incorpore al ordenamiento legal no valdría contra lo expuesto, porque -en el caso de los llamados juegos lícitos, que pueden producir, cuando se cruzan apuestas, efectos legaleslo que hay es simplemente una incorporación, al sistema positivo, de normas de conducta que en sí y por sí no tienen naturaleza jurídica. La conversión de las reglas convencionales sobre juegos en normas de derecho es, pues, algo excepcional, y acontece por obra del legislador. No parece, en consecuencia, plausible buscar en ellas o, concretamente, en las de ajedrez, el modelo explicativo del derecho. Puede, en cambio, partirse de la definición de éste para explicar el caso en que aquéllas, por reconocimiento legal expreso, se transforman en normas de derecho. 
Pero examinemos con mayor cuidado el parangón establecido por Ross. Volvamos, por ejemplo, a su tesis de que el juego de ajedrez puede entenderse como modelo muy simple de lo que se llama un fenómeno social, o a su aserto de que sólo debe hablarse de vida colectiva cuando las conductas individuales se relacionan unas con otras de acuerdo con ciertas reglas. Estas afirmaciones son correctas, como es correcta la de que entre los actos sociales existe una relación análoga a la que, en el juego de ajedrez, se da entre movida y contramovida. Cabe, igualmente, admitir que las reglas de los juegos y las normas del derecho es. tablecen relaciones intersubjetivas. Lo que no es admisible, en cambio, es que toda regla de comportamiento que engendra relaciones de tal jaez sea, eo ipso, jurídica. Pues esas relaciones tienen distinto sentido en el ámbito del derecho y en las esferas de la moral y los convencionalismos. La bilateralidad, característica exclusiva de la regulación jurídica, sólo aparece en las regulaciones sobre juegos cuando éstas han sido incorporadas por el legislador a su propio sistema. Pero es claro que, en tal conyuntura, dejan de ser convencionalismos sociales para convertirse en normas de derecho. Fuera de este caso, aquel atributo no les corresponde. Esto no priva a su regulación del carácter de intersubjetividad señalado por Ross; pero existe una diferencia: mientras en el plano de las normas jurídicas frente al obligado por ellas encontramos siempre un pretensor, en el de los convencionalismos el deber del obligado no es correlativo de un derecho de otro sujeto, sino simplemente de una expectativa o esperanza. En cuanto al deber de no hacer trampas, sobre todo en la hipótesis de juegos en que no median apuestas y la finalidad de los jugadores simplemente es divertirse, ese deber asume tanto índole convencional como carácter ético.

Las dificultades inherentes a la posición de Ross se agudizan cuando éste se pregunta qué debe entenderse por reglas del ajedrez. Recordemos cómo, al contestar su pre- 
gunta, empieza por decir que sólo quiere referirse a las de carácter elemental que prescriben los movimientos de las piezas, la forma de captura, etc., y no a las de la teoría del juego. Las primeras son para él directrices acerca de la forma "en que se debe jugar"; a las segundas las clasifica como reglas técnicas que se expresan por medio de enunciaciones hipotéticas. Tal dicotomía presenta de inmediato un problema, pues entre las reglas de conducta de contenido prescriptivo o atributivo y las llamadas de las artes existe una diferencia esencial. Esta fue claramente precisada por Rodolfo Laun, cuando dijo que, mientras las primeras dan expresión "a un deber condicionado" las otras expresan "una necesidad condicionada". Admitiendo, sin conceder, que el parangón establecido por Ross fuese correcto, resull. taría que las reglas más importantes del juego que sirve de base a sus desarrollos, es decir, las de la teoría del ajedrez, no podrían fungir como pauta explicativa de las normas jurídicas, porque, aun cuando las últimas tienen también estructura hipotética, en todo caso poseen significación normativa, lo que no puede aseverarse de las reglas técnicas. Es cierto que la aplicación de éstas es a veces objeto de un deber, pero ello no dimana de la naturaleza de dichas reglas, sino de la norma que, en tales o cuales condiciones, obliga a un sujeto -0 a determinada clase de sujetos- a darles aplicación. Piénsese, por ejemplo, en los preceptos que obligan a un trabajador a aplicar, para el cumplimiento de las tareas que le impone su contrato de trabajo, las reglas técnicas de su oficio.

Nuevas dificultades surgen cuando recordamos las afirmaciones de Ross acerca de la forma en que es posible establecer las reglas del ajedrez. Aun cuando declara que cabría tomar como base los reglamentos que se observan en los congresos de ajedrecistas o las directrices que ofrecen los tratados sobre el juego, de inmediato añade que esto sería insuficiente, "pues no siempre se adoptan las mismas reglas". No queda entonces más remedio que referir la 
cuestión a las que regulan una partida determinada entre sujetos determinados. Mas para saber cuáles sean éstas resulta indispensable investigar, en cada caso, por cuáles se sienten vinculados los jugadores. Imagínese a qué consecuencias llegaríamos si hiciésemos lo mismo para establecer qué preceptos jurídicos están en vigor. Tratándose de un contrato, por ejemplo, no bastaría inquirir qué normas señalan las consecuencias del negocio; sería preciso, además, preguntar a los contratantes: ¿se sienten Uds. obligados por esas disposiciones? De ser correcto el paralelo que el jurista escandinavo formula habría que descartar - como desprovistas de vigencia - las que a dichos sujetos no les pareciesen "vinculantes". Y algo peor aún: tendría que negarse que esos preceptos fuesen jurídicos.

Sabemos ya que, para Ross, el derecho está en parte constituido por "fenómenos" y, en parte, por "normas", en correlación reciproca. Ahora bien: las segundas son los "esquemas interpretativos" que permiten concebir, como "fenómenos jurídicos", determinadas acciones humanas. La relación entre las normas y esos fenómenos es análoga, en el sentir del jurista escandinavo, a la que existe entre las reglas de ajedrez y ciertos hechos que, a la luz de éstas, son interpretados como "movidas" dentro del juego. De aquí concluye nuestro autor que la expresión "derecho vigente" designa el conjunto de ideas normativas que fungen como sistema de interpretación de fenómenos jurídicos concretos, cuando las normas que los rigen son efectivamente observadas, y quienes las observan se sienten vinculados por ellas. En conexión con este punto, Ross formula dos cuestiones que le parecen esenciales: $1^{a}$ ) ¿De qué modo podemos distinguir determinado conjunto de normas conocido con el nombre de derecho nacional, de otros conjuntos normativos como las reglas del ajedrez o las de cualquiera otro juego? $2^{\mathfrak{a}}$ ) ¿En qué forma se adapta al derecho el concepto de vigencia, si la de un sistema de normas signi. 
fica que éste puede, en virtud de su eficacia, fungir como sistema interpretativo?

En respuesta al primero de los dos interrogantes afirma que expresiones como "derecho sueco", "derecho danés", etc., designan conjuntos individuales de normas comparables a esos otros conjuntos que llamamos "reglas de los juegos". Este aserto le parece suficiente para añadir, con toda tranquilidad, que el problema de la definición del derecho no puede siquiera plantearse, y que las interminables polémicas sobre la naturaleza del orden jurídico tienen su origen en la creencia de que éste deriva su fuerza obligatoria "de una idea a priori”. El error está, prosigue, en olvidar que los llamados "sistemas normativos" son "hechos", y no otra cosa. Parece claro, en consecuencia, que, si ostentan tal carácter, podremos describirlos, teniendo buen cuidado, al hacer su descripción, de no confundir la actitud teóricodescriptiva con la completamente distinta que se traduce en la aprobación o reprobación morales de tal o cual orden concreto. La cuestión básica, para el jurista, consiste, de acuerdo con Ross, en saber cómo es posible distinguir (en lo que al contenido atañe) un sistema jurídico individual de otros conjuntos individuales de normas. Así como, en el caso de las reglas del ajedrez, la coherencia de las mismas depende de que todas ellas se refieren, directa o indirectamente, a lo que deben hacer los jugadores, del mismo modo, en el caso del derecho, sus normas se encuentran todas referidas a acciones $u$ omisiones de determinadas personas. ¿Quienes son éstas, y de qué clase de conductas se trata?

$\mathrm{Al}$ examinar el elemento material de las normas jurídicas observamos, dice Ross, que pueden, desde tal punto de vista, dividirse en dos grandes grupos. Uno está formado por las normas de comportamiento; el otro, por las de competencia. Si partimos de esta dicotomía, caeremos en la cuenta de que, más que hallarse dirigidas a los particulares, las normas del derecho en realidad se dirigen a los órganos de la jurisdicción. Su contenido consiste, bien vistas las 
cosas, en un conjunto de directivas para los tribunales, pues las referidas a los sujetos privados son simples "derivaciones" de las otras. Y como el Estado, al menos en nuestra época, detenta el monopolio de la coacción y, en uso del mismo, puede imponer por la fuerza las decisiones judiciales, cabe afirmar que "un sistema jurídico nacional es el conjunto de reglas relativas a la organización y funcionamiento del poder coactivo estatal". Esta conclusión revela que "los fenómenos jurídicos", como "equivalente" de las normas, no son otra cosa que las sentencias de los jueces. Y tales fenómenos, esto es, los de aplicación del derecho, resultan decisivos para establecer la "vigencia" de las normas aplicadas. El mencionado atributo está, en efecto, condicionado por dos elementos: en primer lugar, por la "eficacia" de las normas, que inequívocamente se manifiesta en que los tribunales las aplican; y, en segundo término, por un factor interno no menos importante: el que consiste en que los órganos de la jurisdicción se sientan "vincula. dos" por ellas.

La aserción de que una norma es derecho vigente supone, de acuerdo con Ross, que media una correspondencia entre el sistema a que la norma pertenece y cierta realidad social, que el propio Ross hace consistir en la aplicación de tal norma por los tribunales, lo que implica, al mismo tiempo, que los jueces tienen la convicción de que aquélla los vincula. Ross considera que esta aserción sólo es correcta si podemos someterla al principio de verificación. Las proposiciones concernientes al derecho vigente quedan verificadas cuando, cumplidas ciertas condiciones, las normas de que se trata soll aplicadas por los jueces. Las condiciones a que Ross alude son éstas: a) los asertos acerca del derecho vigente en cierto momento y lugar no se refieren al pasado; b) tampoco pueden referirse a un futuro muy lejano.

Los mencionados asertos no tienen un carácter absoluto; en rigor son "predicciones" sobre lo que los tribunales 
harán en determinadas circunstancias. Dicho de otro modo: la realización de semejantes predicciones es solamente probable, y la probabilidad de que se cumplan depende del material empírico utilizado por quien trata de adelantarse al futuro. "La probabilidad es fuerte y la norma posee un alto grado de vigencia, si la previsión se apoya en una doctrina sólida, sostenida por una larga serie de precedentes no discutidos, o se basa en una disposición legislativa cuya interpretación ha confirmado una práctica duradera y coherente. Por el contrario, la probabilidad es débil y, en consecuencia, la norma tiene un bajo grado de vigencia, si la previsión se ha hecho de acuerdo con un precedente dudoso y único, o deriva de 'la razón' o de ciertos 'principios" " (44).

De acuerdo con los anteriores desarrollos habría que decir que las leyes que el legislador expide y que aún no han sido aplicadas por los tribunales, no son normas jurídicas, porque, ni tienen eficacia, ni han condicionado en ninguna forma la actividad de los encargados de la función jurisdiccional. Además, antes de los actos de aplicación, no puede saberse, relativamente a ellas, si los jueces se sienten o no ligados por las mismas. Por otra parte, es indudable que el legislador no pretende, al promulgarlas, actuar como profeta, es decir, hacer predicciones sobre la conducta futura de aquéllos. Lo que los órganos legislativos se proponen es, ante todo, regular la conducta de los particulares y, en segundo término, establecer qué consecuencias sancionadoras deberán atribuirse a los actos violatorios de esa regulación. El sentido de la tarea legislativa no es, en ningún caso, trazar esquemas o pautas de un actuar probable, sino enlazar en forma lógicamente necesaria, a los hechos que realicen los supuestos legales, el nacimiento de determinados deberes y facultades. Estos deberes $y$ facultades no se refieren solamente, como es obvio, a la conducta de los jueces, sino al comportamiento de los particulares. El legislador no hace nunca depender, de la aquiescencia de los 
destinatarios, o del hecho de que se sientan "vinculados", la fuerza obligatoria de las leyes. Si aceptamos, lo que desde el punto de vista lógico no encierra contradicción, que una norma puede, de hecho, ser cumplida siempre por los que en todo caso podrían violarla, tendríamos que llegar a la conclusión, de acuerdo con la tesis de Ross, de que tal norma no tendría vigencia, porque no se daría el caso de que los tribunales tuvieran que aplicarla.

Como lo ha mostrado Eugenio Bulygin en su artículo "El concepto de vigencia de Alf Ross" (Revista del Colegio de Abogados de La Plata, 1963), el jurista escandinavo da dos respuestas diferentes a la pregunta sobre qué debe entenderse por aplicación de una norma. Por una parte sostiene que la simple observación de la conducta externa del juez no es suficiente para determinar qué normas aplica éste, pues para que pueda hablarse de aplicación es indispensable que el órgano jurisdiccional se sienta vinculado por los preceptos que son objeto del acto aplicador. En otro pasaje declara, en cambio, que la aplicación de un precepto jurídico sólo puede significar que en las decisiones en que se dan por probados los hechos condicionantes que la norma señala, ésta es "parte esencial" del razonamiento que funda la sentencia, lo que convierte a dicha norma en factor determinante de la conclusión a que se llega. De acuerdo con el mencionado pasaje, observa Bulygin, "no es en modo alguno necesario que el juez se sienta vinculado por la norma o la viva como socialmente obligatoria; basta con que la invoque para fundamentar su fallo" (Artículo citado, pág. 10). Si bien es cierto, prosigue Bulygin, que por regla general los jueces se sienten obligados por el derecho y fundan sus sentencias en normas que consideran obligatorias, ello no ocurre de modo indefectible. Puede, en efecto, darse el caso, mencionado por Ross, de que los jueces apliquen, por temor, un orden jurídico que a sus ojos no tiene más apoyo que la fuerza de quienes pretenden imponerlo, como ocurre, verbigracia, cuando ese orden proviene de una au- 
toridad militar en un pais ocupado. ¿Negaremos, en tal hipótesis, la índole jurídica de las disposiciones aplicadas? Bulygin opina que "la caracterización de la vigencia mediante el requisito del sentimiento de obligatoriedad social resulta no sólo inadecuada para la descripción de ciertas normas, generalmente consideradas jurídicas, sino que incluso conduce a contradicciones dentro de la teoría de Ross. La segunda respuesta, en cambio, hace innecesario practicar la introspección judicial y permite hablar de normas jurídicas vigentes, aunque los jueces actúen motivados por temor o por el deseo de favorecer a una de las partes, ya que no hace referencia a los sentimientos del juez, sino a los fundamentos de la sentencia. No interesa qué siente el juez, sólo importa lo que dice. Una norma es vigente cuando el juez la usa para justificar su decisión (cualquiera sean los motivos que lo impulsan a tomarla)" (Artículo citado, pág. 11).

Aun cuando concedamos nuestra preferencia al segundo de estos dos puntos de vista, es indudable que tiene el defecto de referir exclusivamente el problema a las resoluciones judiciales. Por lo demás, el hecho de que los jueces funden sus fallos en normas jurídicas, es buena prueba de la prioridad lógica de los fundamentos aducidos $\mathrm{y}$, por ende, de que la vigencia de las normas aplicadas no deriva del hecho de la aplicación, ya que ésta presupone que las normas en que se basa están en vigor.

Que Ross tiene conciencia de todo esto lo demuestran, en nuestro sentir, tanto la discusión que hace del realismo psicológico y del realismo conductista, como la conclusión ecléctica adoptada por él. Por ello asevera que ninguna de esas posiciones puede, aisladamente, resolver el problema de la vigencia, y que ésta sólo se explica a través de una síntesis de las dos posturas.

Conviene subrayar el hecho de que, si bien los defensores de ambas están convencidos de que el orden jurídico puede y debe ser explicado descriptivamente, por la natu- 
raleza puramente fáctica que le atribuyen, ninguno de los partidarios del realismo, en sus dos versiones básicas, puede prescindir, pese a sus esfuerzos, de los conceptos tradicionales de norma, deber jurídico y derecho subjetivo, ni liberarse por completo de las significaciones normativas que los mismos contienen. 
In this article the author discusses Ross's conception of law. The first objection arises in connection with the use made of the term directives in Ross's book On Law and Justice. The Scandinavian jurist gives this name to utterances with no representative meaning made in order to influence another's behaviour. When the generic term utterance refers to norms of conduct, it seems to suggest, from the beginning, that normative judgments, far from imposing duties or granting rights merely express the desire to influence another in such and such a way, not because he must act thus or has the right to do so, but because whoever makes this utterance wishes to affect in some way the others behaviour. Norms cannot be put on the same level as mandates, petitions, suggestions and desires, since there are no reasons to maintain that when the legislator makes a law or a court of law passes sentence, they are doing something which may be be compared to the formulation of a desire or to the expression of a demand.

Neither is the author of this article in agreement with Ross's thesis on the interpretation that must be put on the sentences which appear in a textbook of law, for example in treatises on civil or administrative law. If, in a manual of Mexican Civil Law, we should find the words: "He who discovers treasure in another's land has the right to keep for himself half of the treasure, and is obliged to surrender the other half to the owner of the property", such words clearly have no descriptive sense, since they do not describe the conduct of people who have discovered treasure, nor do they hypothetically describe the conduct of all those who may have that good fortune. The judgment expressed by that sentence is in one sense atributive of a right and in another, impositive of a duty. Therefore, one is not dealing with an assertions, since the words which make it up do not express, as Husserl would say, a matter of fact, nor do they express the actual behaviour of those who discover treasure. If, on the other hand, we say: "In accordance with article 877 of the Civil Code of the District and of Federal Territories, 'he who discovers treasure on another's land has the right to keep for himself half of the treasure, and is obliged to surrender the other half to the owner of the property" ", this expression will have the force of an assertion. But it is one thing to declare that an article in a code says such and such, and quite another to express the norm contained in that article. 
The third question is this: can we really consider chess, or any other game as a "model" or "explanatory pattern" of the legal order?

The rules of games cannot be used as the starting point for a doctrine of what law is, because they do not generally come within the scope of the legal, but within that of social conventions. Even when the legislator occasionally incorporates those rules into his system, transforming them into legal precepts, such a conversion is somewhat exceptional and is only brought about by a legislative decision.

Ross argues that the game of chess may be taken as a very simple model of what is called social phenomenon, and maintains that one should only talk of community life when individual behaviour is inter-related according to certain rules. There is no doubt that the norms of law establish inter-subjective relations to the same extent as the rules of a game. What is doubtful, however, is the assertion that any pattern of behaviour which leads to relations of that kind should be, eo ipso, legal. For these relations have a different significance in the sphere of law than in that of morals and social conventions. Bilateralness, which is an exclusive characteristic of the legal norms, is encountered in the rules of games only when the legislator has incorporated those rules into the system of laws in force. In that eventuality, however, they cease to be social conventions and become norms of law. This does not rid them of the quality of inter-subjectivity outlined by Ross, but whilst in the field of legal norms we always encounter a claimant and an obligor, in that of social conventions the duty of the obligor is not a co-relative of the right of another person, but simply of an expectation or hope.

Ross says that when he speaks of the rules of chess he only wishes to refer to those of an elementary nature which prescribe the movement of the pieces, the form of capture, etc., and not to those which govern the theory of the game. For him the first are directives on the way in which one should play; he classifies the second as technical rules which are expressed in hypothetical statement. This dicthotomy presents a problem, for there is a fundamental difference between the rules of conduct, which are either prescriptive or attributive in content, and the rules of art. However, the first express, in the terms of Rudolf Laun, "a conditioned duty"; the other, "a conditioned necessity". If we allow the comparison used by Ross, the most important rules of the game, that is the theory of chess, which serve as a base for its developments, cannot work in the same way as an explanatory pattern of 
legal precepts, because, even when these precepts too have a hypothetical structure, in each case they have an implicit normative significance which is fundamental to them.

New difficulties arise when we recall what Ross has written on the way in which the rules of that game can be established. As the same ones are not always adopted, the question has to be referred to those rules which govern a particular game between particular persons. But for us to know which these may be, it becomes essential to investigate in each case which ones the players feel bound by. What would happen if we did the same thing in order to establish which legal precepts were in force? When dealing for example with a contract, it would not be sufficient to inquire into the normative consequences of the transaction; one would also have to ask the contractors: "Do you consider yourselves bound by these consequences?"

The relation between norms and legal phenomenon is analogous, according to Ross, with that between the rules of chess and certain actions which, in the light of the rules, are interpreted as moves within the game. From this he concludes that the expression "valid law" refers to the normative ideas as a whole, which act as a system of interpretation for concrete legal phenomena, when the norms which govern them are in fact observed, and those who observe them feel bound by them.

Ross says that expressions like "Swedish law", "Danish law", etc., refer to individual set of norms comparable with those called "the rule of games". The mistake made by many jurists, Ross continues, is that they forget that what are termed "normative systems" are "facts" and nothing else. In describing these facts one must be very careful not to confuse the theoretical - descriptive attitude with the completely different one which is manifested in moral approval or disapproval of a particular established system. According to Ross the basic question for a jurist is how one can distinguish one individual legal system from other individual sets of norms. In the case of law its norms all refer to actions or omissions by particular people. Who are they, and with what sort of conduct are we dealing?

Ross divides legal precepts into norms of conduct and norms of competence and he maintains that such precepts, rather than referring to individuals, refer in fact to the organs of jurisdiction. He concludes from this that "legal phenomena", as "equivalent" to norms, are nothing more than the sentences of the judges. In other words, the assertion that a norm is valid law presupposes that there is some correspondence between the system to which the norm 
belongs and a certain social reality, the application of a particular norm by courts of law insofar as they fell bound by it. Further, in order that the above assertion may be verified, it must fulfill two conditions: a) Thes statemnets concerning valid law at the present moment should not refer to the past; b) neither should they refer to the far distant future. Such statements are not absolute; rather they are "predictions" of what courts of law will do in certain circumstances.

If we accept these ideas we will have to say that these laws drawn up by the legislator which have not yet been applied by the courts are not legal norms, because they have no efficacy, nor have they affected in any way the actions of those responsible for the working of the legal system. Moreover, before their practical application, one cannot know whether or not the judges will feel themselves bound by the laws. On the other hand, it is certain that when he publishes them the legislator does not pretend to be a prophet, that is, to make predictions on the future conduct of the judges. The point of such a legislative task is not to sketch pro. bable actions, but to link up, in the necessary logical form, the facts which fulfill the legal suppositions to certain rights and duties. And these do not refer only to the conduct of the judges but also to the behaviour of individuals.

As Eugenio Bulygin showed in his article "The Concept of Valid Law in Alf Ross" (Revista del Colegio de Abogados de la Plata, 1963), the Scandinavian jurist gives two different replies to the question of what should be understood by the application of a norm. At one point he maintans that merely to observe the external conduct of the judge is not enough for us to determine which norms he is applying, since we do not know if he feels bound by the precepts which are the object of the act of application. But in another passage he declares that the application of a legal precept can only mean that, in decisions in which the conditioning facts indicated by the norms are taken as proved, this is the "essential part" of the reasoning on which the sentence is based, thus con. verting that norm into a determining factor of the conclusion which is reached. But then, Bulygin observes, "it is in no way necessary that the judge should feel bound by the norm or that he should live according to it as a social obligation; it is sufficient that he should invoke it as a foundation for his decision" (Page 10 of the said article). Bulygin says that "by virtue of the neccessity of a feeling of social obligation, Ross's interpretation of valid law becomes not only inadequate for the discussion of certain norms, generally considered legal, but it also leads to contradictions within 
Ross's theories. His second answer, on the other hand, makes it unnecessary to indulge in judicial introspection, and permits one to talk of legal norms in force, even though the judges may be motivated by fear or the desire to favour one of the parties; thus making no reference to the feelings of the judge but rather to the foundation of the sentence", (Page 11 of the said article). Even when we say that the second of these opinions is preferable, it clearly has the defect of restricting the problem entirely to legal decisions. For the rest, - the fact that the judges base their sentences on legal norms - is a good proof of the logical priority of the adduced reasons, and consequently a proof that the validity of the applied norms does not derive from the fact of their application, because the latter presupposes that the norms on which they are based are valid law.

That Ross is aware of all this is demonstrated, in our opinion, as much by his discussion of psychological realism and behaviouristic realism as by the ecletic conclusion which he draws. In this conclusion he asserts that none of those doctrines can in isola. tion resolve the problem of legal validity, and that this can only be explained by means of a synthesis of the two views.

It is useful to stress the fact that though supporters of both may be convinced, by virtue of the purely factual nature which they attribute to it, that the legal system can and must be explained discriptively, none of the adherents of realism, in its two basic versions, can dispense, try as they might, with the traditional concepts of norm, right and legal duty nor can they free themselves completely of the normative associations which they contain. 